\title{
A difficult to diagnose case of low-grade endometrial stromal sarcoma with smooth muscle differentiation treated with laparoscopic surgery: A case report
}

\author{
TOMOYUKI ICHIMURA, MARI KASAI, KENJI IMAI, MAKOTO YAMAUCHI, \\ TAKESHI FUKUDA, TOMOYO YASUI and TOSHIYUKI SUMI
}

Department of Obstetrics and Gynecology, Osaka City University Graduate School of Medicine, Osaka 545-8585, Japan

Received November 8, 2021; Accepted January 19, 2022

DOI: $10.3892 / \mathrm{mco} .2022 .2525$

\begin{abstract}
Low-grade endometrial stromal sarcoma (LGESS) is a rare uterine tumor, accounting for $<1 \%$ of all uterine cancer cases. LGESS has several variations and the frequency of tumors exhibiting smooth muscle differentiation is $10-30 \%$ of LGESS cases, making such cases even rarer. The present report described the case of a patient with LGESS with smooth muscle differentiation, who was diagnosed as having uterine leiomyoma by preoperative needle biopsy and then underwent laparoscopic surgery. The patient was a 41-year-old woman. MRI findings revealed a diffusely hyperintense uterine tumor on T2-weighted images, thus needle biopsy was performed. This tumor was initially diagnosed as leiomyoma, due to the pathological findings of the biopsied specimen, which possessed tumor cells with spindle-shaped nuclei arranged in a cord and positive immunostaining for smooth muscle actin. The patient was subsequently followed up, and MRI findings after 29 months showed tumor growth. Needle biopsy was performed again and the findings were the same as those of the first biopsy; therefore, this tumor was diagnosed as a leiomyoma and laparoscopic hysterectomy was performed. However, the pathological findings of the excised uterus showed small round tumor cells and CD10 immunostaining positivity, thus the tumor was finally diagnosed as LGESS. The patient requested to be followed up and has shown no signs of recurrence 20 months after surgery. The results of retrospective examination in this case suggested that the presence of regions where only CD10 was positive in immunostaining analysis for SMA and CD10 was useful for needle biopsy diagnosis of LGESS with smooth muscle differentiation.
\end{abstract}

Correspondence to: Dr Tomoyuki Ichimura, Department of Obstetrics and Gynecology, Osaka City University Graduate School of Medicine, 1-4-3 Asahimachi, Abeno-ku, Osaka 545-8585, Japan E-mail: 3912-tomo@med.osaka-cu.ac.jp

Key words: low-grade endometrial stromal sarcoma, smooth muscle differentiation, needle biopsy, CD10 immunostaining, laparoscopic surgery

\section{Introduction}

Uterine sarcoma is a rare mesenchymal tumor that occurs primarily in the uterine corpus, and endometrial stromal sarcoma is an even rarer type of tumor that accounts for $\sim 10 \%$ of uterine sarcomas (1). Endometrial stromal sarcoma is classified into high-grade endometrial stromal sarcoma (HGESS) and low-grade endometrial stromal sarcoma (LGESS) according to histological characteristics. The tumor cells of HGESS cases show marked nuclear atypia and increased mitosis. On the other hand, nuclear atypia is mild or absent, and mitotic figures are rarely seen in LGESS cases. LGESS may be difficult to distinguish from uterine leiomyoma by MRI examination, as they are both mesenchymal tumors, especially in the case of degenerative leiomyoma. Therefore, there are reports of LGESS cases diagnosed by postoperative pathological examination after surgery performed under a preoperative diagnosis of uterine leiomyomas (2). The histopathological findings of LGESS are characterized by the proliferation of small tumor cells with round to oval nuclei, similar to proliferative endometrial stromal cells, and tumor cells are immunohistochemically positive for CD10. These findings usually make it easy to histologically distinguish between LGESS and uterine leiomyoma that are usually immunohistochemically positive for SMA and negative for CD10. However, LGESS with smooth muscle differentiation is morphologically and immunohistochemically similar to leiomyoma; thus it is difficult to distinguish it from uterine leiomyoma when only the region showing differentiation is observed. The present report describes the case of LGESS with smooth muscle differentiation that was diagnosed as uterine leiomyoma by preoperative needle biopsy and treated with laparoscopic surgery. The possibility of preoperative diagnosis of similar cases with reference to the findings obtained from retrospective immunohistochemical studies in this case was also discussed.

\section{Case report}

The patient was a 41-year-old, gravida 1, para 1 female. She visited a nearby clinic with complaints of ovulation bleeding and lower abdominal pain, and was referred to Osaka City University hospital due to the presence of a $31 \times 21 \mathrm{~mm}$ uterine body mass with an internal hyperechoic region, as identified by 
transvaginal ultrasonography. MRI findings showed a diffuse high signal of a $\sim 30 \mathrm{~mm}$ uterine corpus tumor on T2-weighted images, but a low signal on T1-weighted images (Fig. 1A and B). In addition, since the diffusion-weighted image showed a high signal, but the apparent diffusion coefficient did not decrease, the tumor was not restricted in diffusion and was considered to have few findings suggestive of malignant disease. The protocol for MRI imaging is as follows: TR (time to repeat); 4,000 milliseconds, TE (echo time); 85 milliseconds (Sagittal)/100 milliseconds (Axial), receive bandwidth; $100 \mathrm{~Hz}$, Field of view; $27 \mathrm{cn}$, Slice thickness; $5 \mathrm{~mm}$, Matrix number; $512 \times 273$, b-value; 0-1,000 second $/ \mathrm{mm}^{2}$, diffusion measurement time; 108. Blood analysis showed no abnormalities in serum lactate dehydrogenase and CA125 levels. Based on the aforementioned results, it was considered that there were few findings supportive of uterine leiomyosarcoma, and the MRI findings were considered to be different to that of typical uterine leiomyomas. Therefore, it was decided to perform a histological examination by transcervical needle biopsy (needle biopsy).

Needle biopsy was performed at the lithotomy position under transvaginal ultrasound guidance. A puncture attachment was attached to the transvaginal probe, and a tru-cut type 17-gauge $250 \mathrm{~mm}$ puncture needle was inserted into the tumor to collect the specimen. The maximum size of the tissue collected by the biopsy needle was $1 \times 17 \mathrm{~mm}$ per tissue, and a total of three specimen were collected. The findings of the H\&E-stained specimen of the tissue obtained by needle biopsy showed that tumor cells with spindle nuclei without atypia were arranged in a cord (Fig. 2A), and the immunostaining analysis findings were positive for smooth muscle actin (SMA) (Fig. 2B). The protocols for H\&E staining and immunostaining are as follows: H\&E staining protocol; i) Deparaffinize (dunk for $10 \mathrm{~min}$ in xylene, 3 tanks). ii) Remove xylene (dunk for 5 min in 100\% ethanol, 3 tanks). iii) Flooding (dunk for 5 min in $95 \%$ and $70 \%$ ethanol). iv) Wash with water and distilled water. v) Hematoxylin stain (dunk for 4 min in Hematoxylin stain). vi) Wash with flowing tap water. vii) Eosin stain (dunk for $2 \mathrm{~min}$ in Eosin stain). viii) Dehydration (dunk for $30 \mathrm{sec}$ in 70 and $95 \%$ ethanol, then dunk for $5 \mathrm{~min}$ in $100 \%$ ethanol, 3 tanks). ix) Clear (dunk for $10 \mathrm{~min}$ in xylene, 3 tanks), Immunostaining protocol; i) Preheat the antigen retrieval buffer $(100 \mathrm{mM}$ Tris, $\mathrm{pH} 9.5)$ to $95^{\circ} \mathrm{C}$. ii) Wash tissues in PBS three times for $5 \mathrm{~min}$. iii) Incubate the tissues for $20 \mathrm{~min}$ with PBS containing Triton X-100. iv) Wash tissues in PBS three times for $5 \mathrm{~min}$. v) Incubate tissues with $1 \% \mathrm{BSA}$ for $30 \mathrm{~min}$. vi) Incubate tissues in the diluted primary antibody in $1 \%$ bovine serum albumin (BSA) in a humidified chamber for $1 \mathrm{~h}$ at room temperature. vii) Decant the solution and wash the tissues three times in phosphate-buffered saline (PBS), $5 \mathrm{~min}$ each wash. Based on these results, the tumor was diagnosed as a uterine leiomyoma, and was followed up as she had no subjective symptoms.

Ultrasonography performed 29 months after the initial visit showed the appearance of cystic lesions on both sides of the uterus. The uterine tumor showed a gradual tendency for growth, and it measured $\sim 60 \mathrm{~mm}$ in size at this point. Therefore, MRI examination was performed again. Cystic lesions of $52 \times 40 \mathrm{~mm}$ on the right dorsal side of the uterus and $36 \times 28 \mathrm{~mm}$ on the left dorsal side of the uterus were observed, both showing an equal signal intensity on T2-weighted images and a high signal intensity on T1-weighted images, after performing fat suppression. Bilateral endometriotic ovarian cysts were suspected from the MRI results. Conversely, regarding the uterine corpus tumor, although the size had increased to $68 \times 55 \mathrm{~mm}$, it was considered that the T1-weighted image showed a low signal intensity and was not subject to diffusion restriction as before, and there was no change in the internal properties (Fig. 3A-D).

She was recommended for surgery for the bilateral ovarian cysts, and she also requested surgery for the uterine tumor due to her hypermenorrhea. Blood tests were performed on this case prior to the first needle biopsy, the second needle biopsy and surgery, but no specific findings were found in the results of blood analysis including tumor markers except for anemia before needle biopsy (Table I). Therefore, it was decided to perform laparoscopic surgery after histological examination for the uterine tumor by a second needle biopsy. The second needle biopsy was performed at the lithotomy position under transabdominal ultrasound guidance. A puncture guide tube was inserted into the uterine cavity transcervically, and the tip of the inserted guide tube was guided under transabdominal ultrasound to the vicinity of the target tumor, and the puncture needle was continuously inserted into the tumor to collect tissue. The pathological diagnosis of the tissue collected by needle biopsy was leiomyoma, as in the first diagnosis (Fig. 4A and B), Based on these results, a laparoscopic simple total hysterectomy, bilateral ovarian cystectomy and bilateral salpingectomy were performed. The removed uterus, bilateral ovarian cysts and bilateral fallopian tubes were placed in a collection bag and delivered transvaginally. The uterus was shredded in a bag. Macroscopic findings of the surgical specimen showed no bleeding inside the uterine corpus tumor and no remarkable findings in the endometrium. On the microscopic image, the tumor had infiltrated the surrounding muscle layer. H\&E staining findings showed a region where tumor cells with spindle-shaped nuclei proliferated and a region where elliptical and relatively small tumor cells proliferated densely in a swirling manner around the arterioles, and the former area was considered to account for $\sim 30 \%$ of the specimen. In the latter area, immunostaining analysis showed extensive positivity for CD10. In addition, no nuclear atypia or mitotic figures were observed in any of the regions, so this tumor was diagnosed as LGESS with smooth muscle differentiation (Fig. 5A-E). Regarding the ovaries, both sides were diagnosed with benign endometriotic cysts. Although additional treatments, such as bilateral salpingo-oophorectomy were offered, consent for additional treatment was not obtained. Therefore, it was decided to follow up after confirming that there was no neoplastic lesion on the image via a computed tomography examination. No lesion was found on the image, and the final diagnosis was LGESS stage IB (pT1bNxM0). No signs of recurrence were observed 20 months after the operation.

\section{Discussion}

LGESS is a rare disease, accounting for $<1 \%$ cases of all uterine malignant tumors; however, it is the second most common malignant mesenchymal tumor of the uterus (3). Amongst the mesenchymal tumors of the uterus, those derived from the 

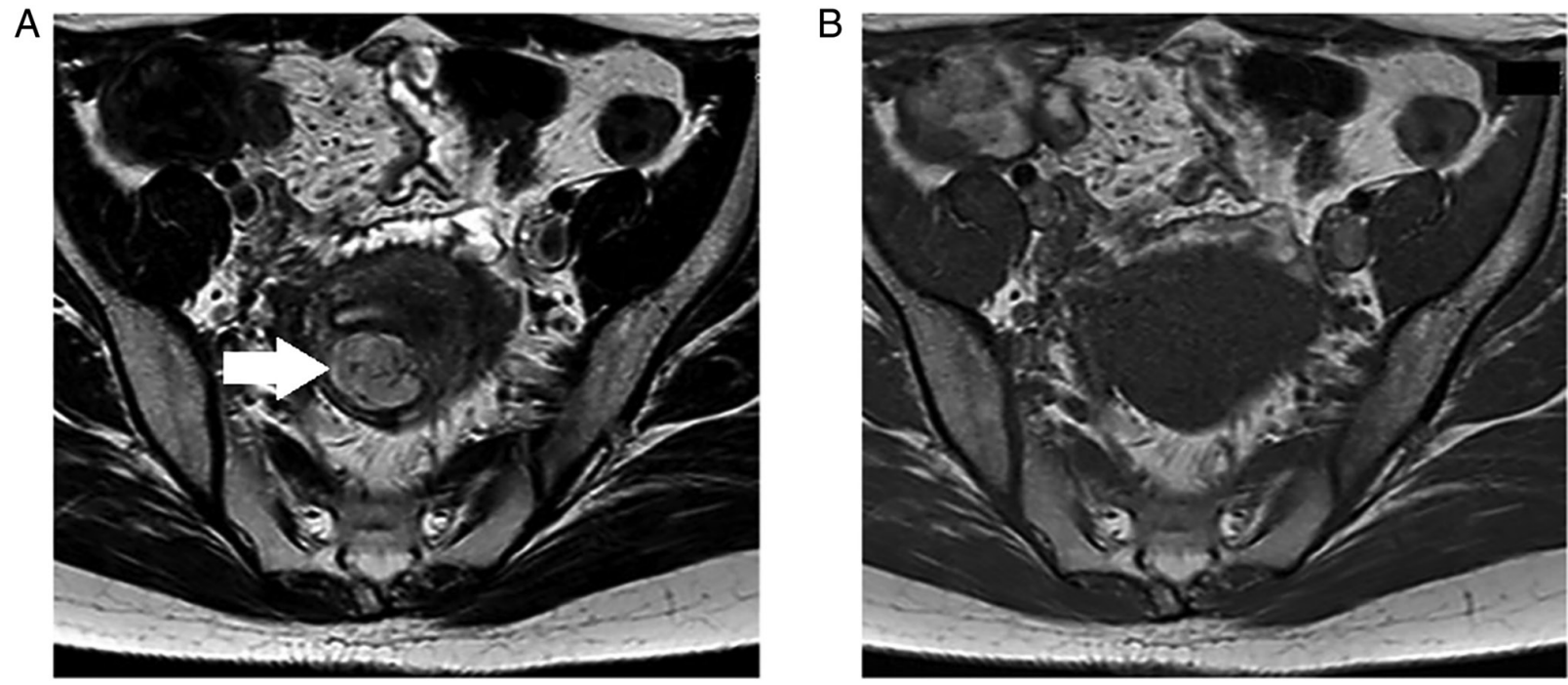

Figure 1. Magnetic resonance imaging scan from the first visit. The uterine tumor found in the muscular layer of the posterior wall measured about $30 \mathrm{~mm}$ in diameter, and showed a diffusely high signal on T2-weighted image and a low signal on T1-weighted image. (A) T2-enhanced axial image. Uterine tumor is indicated by a white arrow. (B) T1-enhanced axial image.
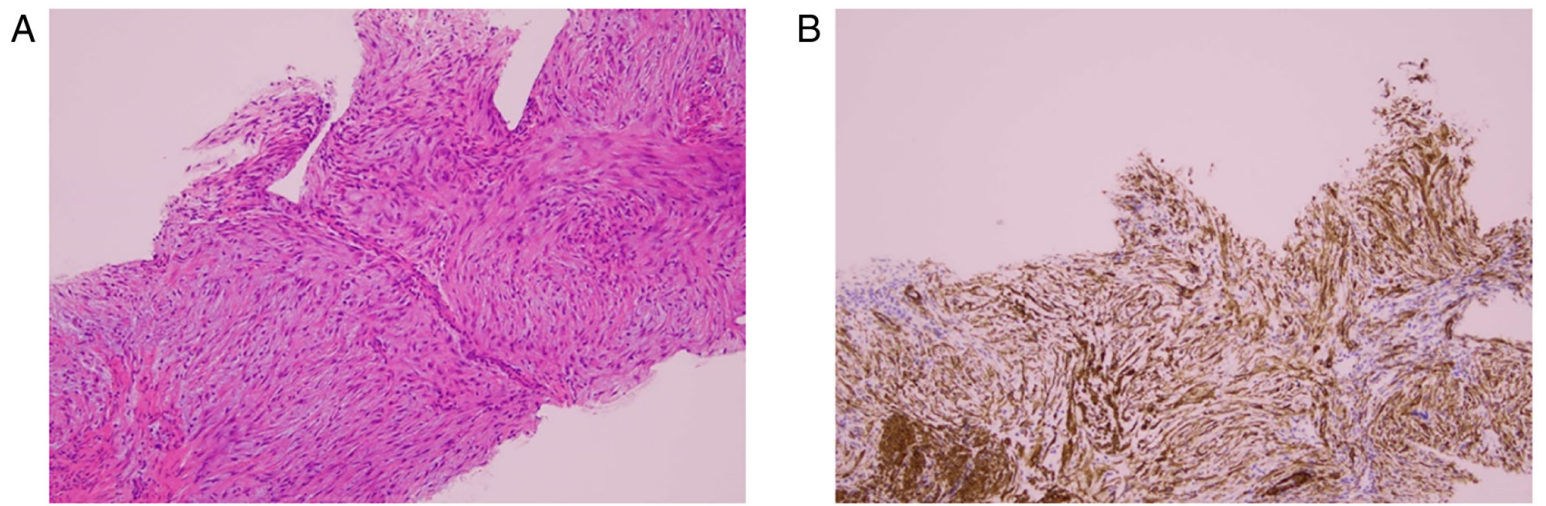

Figure 2. Microscopic image of the first needle-biopsied specimen. (A) H\&E-stained specimen. Tumor cells with spindle-shaped nuclei without any abnormalities were arranged in a cord. (B) SMA-immunostained specimen. SMA is diffusely positive. Magnification, x100. SMA, smooth muscle actin.

endometrial stromal cells include LGESS, HGESS, undifferentiated sarcoma (US) and endometrial stromal nodule (ESN), of which ESN is a benign disease. Histologically, LGESS is characterized by the proliferation of small round tumor cells, similar to the proliferative phase of endometrial stromal cells. Usually, nuclear atypia is absent or mild, and the mitotic index ranges from zero to several/10 high power field (HPF). Although LGESS and ESN are similar in cell properties, they are distinguished by the presence or absence of infiltrative proliferation in the surroundings. Therefore, strictly speaking, it is impossible to distinguish between the two tumors without using an excised uterine specimen containing the tumor. HGESS is also a mesenchymal tumor consisting of cells similar to the proliferative endometrial stromal cells, but unlike LGESS, it exhibits a high degree of nuclear atypia and several necrotic regions. The mitotic figure is conspicuous and generally shows a mitotic index exceeding 10/10 HPF, which is a distinguishing point from LGESS. US is a tumor with strong nuclear atypia, which is difficult to determine as derived from endometrial stromal cells. Characteristically, LGESS is immunohistochemically positive for CD10, which is a distinguishing point from other mesenchymal tumors, such as uterine leiomyoma. However, LGESS can possess several variations, and the frequency of LGESS with smooth muscle differentiation is $10-30 \%$ (4). LGESS with smooth muscle differentiation, especially in cases where the leiomyoma-like region is relatively large, as in this case, when only this region is observed, proliferation of spindle-shaped tumor cells is confirmed and SMA is immunohistochemically positive, thus it becomes difficult to distinguish LGESS from leiomyoma.

A biopsy for mesenchymal tumors of the uterus is not a commonly performed examination, such as the endometrial biopsy for endometrial epithelial tumors. With the approval of the Institutional Review Board and informed consent before examination, we have performed histological examination for uterine mesenchymal tumors by needle biopsy in $\sim 700$ cases between 1994 and the present (5). Since malignant tumors are usually accompanied by nuclear atypia, it can be inferred that the tumor is a malignant tumor, such as sarcoma, by confirming the findings of tumor cell proliferation with atypical nuclei in needle biopsy specimen. Furthermore, since the presence of necrosis is a characteristic finding of 
Table I. Blood test results.

A, Results of blood test performed before the first needle biopsy $^{\mathrm{a}}$

\begin{tabular}{lc}
\hline Inspection item & Result (normal range) \\
\hline White blood cells & $4,100 / \mu \mathrm{l}(4,300-8,000)$ \\
Hemoglobin & $10.8 \mathrm{~g} / \mathrm{dl}(11.3-14.9)$ \\
Platelets & $26.5 / \mathrm{x} 10,000 \mu 1(18.0-34.0)$ \\
Blood urea nitrogen & $10 \mathrm{mg} / \mathrm{dl}(8-20)$ \\
Creatinine & $0.63 \mathrm{mg} / \mathrm{dl}(0.40-0.90)$ \\
Aspartate amino-transferase & $14 \mathrm{U} / 1(13-30)$ \\
Alanine amino-transferase & $10 \mathrm{U} / 1(6-27)$ \\
Total bilirubin & $0.4 \mathrm{mg} / \mathrm{dl}(0.2-1.0)$ \\
Lactate dehydrogenase & $179 \mathrm{U} / \mathrm{l}(124-222)$ \\
C-reactive protein & $0.21 \mathrm{mg} / \mathrm{dl}(0-0.40)$ \\
\hline
\end{tabular}

B, Results of blood test performed before the second needle biopsy $^{\mathrm{a}}$

\begin{tabular}{lc}
\hline Inspection item & Result (normal range) \\
\hline White blood cells & $6,300 / \mu \mathrm{l}(4,300-8,000)$ \\
Hemoglobin & $10.6 \mathrm{~g} / \mathrm{dl}(11.3-14.9)$ \\
Platelets & $27.0 / \mathrm{x} 10,000 \mu 1(18.0-34.0)$ \\
Blood urea nitrogen & $11 \mathrm{mg} / \mathrm{dl}(8-20)$ \\
Creatinine & $0.67 \mathrm{mg} / \mathrm{dl}(0.40-0.90)$ \\
Aspartate amino-transferase & $14 \mathrm{U} / 1(13-30)$ \\
Alanine amino-transferase & $11 \mathrm{U} / 1(6-27)$ \\
Total bilirubin & $0.3 \mathrm{mg} / \mathrm{dl}(0.2-1.0)$ \\
Lactate dehydrogenase & $170 \mathrm{U} / \mathrm{l}(124-222)$ \\
C-reactive protein & $0.05 \mathrm{mg} / \mathrm{dl}(0-0.40)$ \\
\hline
\end{tabular}

C, Preoperative blood test results ${ }^{\mathrm{b}}$

\begin{tabular}{lc}
\hline Inspection item & Result (normal range) \\
\hline White blood cells & $4,400 / \mu 1(4,300-8,000)$ \\
Hemoglobin & $12.3 \mathrm{~g} / \mathrm{dl}(11.3-14.9)$ \\
Platelets & $21.8 / \mathrm{x} 10,000 \mu 1(18.0-34.0)$ \\
Blood urea nitrogen & $12 \mathrm{mg} / \mathrm{dl}(8-20)$ \\
Creatinine & $0.60 \mathrm{mg} / \mathrm{dl}(0.40-0.90)$ \\
Aspartate amino-transferase & $15 \mathrm{U} / \mathrm{l}(13-30)$ \\
Alanine amino-transferase & $11 \mathrm{U} / 1(6-27)$ \\
Total bilirubin & $0.4 \mathrm{mg} / \mathrm{dl}(0.2-1.0)$ \\
Lactate dehydrogenase & $200 \mathrm{U} / \mathrm{l}(124-222)$ \\
C-reactive protein & $0.04 \mathrm{mg} / \mathrm{dl}(0-0.40)$ \\
Cancer antigen 125 & $22 \mathrm{U} / \mathrm{ml}(0-35)$ \\
Carbohydrate antigen $19-9$ & $17 \mathrm{U} / \mathrm{ml}(0-37)$ \\
Carcinoembryonic antigen & $2.7 \mathrm{ng} / \mathrm{ml}(0-5.0)$
\end{tabular}

${ }^{a}$ No special findings other than mild anemia; ball were within normal range, including the values of tumor markers.

sarcoma, it can be determined that the tumor is a sarcoma by confirming the coagulative tumor cell necrosis region with a needle biopsy specimen. Conversely, needle biopsy is not useful in diagnosing tumors with few nuclear atypia and without regions of necrosis, and LGESS is one such tumor that is difficult to diagnose with a needle biopsy. However, needle biopsies have been performed on 4 previous cases of LGESS, and the possibility of LGESS from the pathological results in all cases is considered. The reason why LGESS could be suspected on needle biopsy specimens is that the growth of small round tumor cells with few abnormalities was observed, and that the tumor cells showed positive immunostaining for CD10. These two findings are also characteristic of ESN, and LGESS and ESN are distinguished by the presence or absence of infiltrative growth into the surroundings. Therefore, needle biopsy alone cannot distinguish between the two tumors, and it does not lead to a definitive diagnosis of LGESS. However, needle biopsy can at least predict the possibility of LGESS, and distinguish it from degenerative leiomyoma. The pathological results of the two needle biopsies of this case were both judged to be leiomyoma. This was due to the fact that LGESS with smooth muscle differentiation shows the same H\&E staining and immunostaining findings as leiomyoma depending on the site where the tissue was collected. The region showing differentiation in this case was $\sim 30 \%$. Although it was considered a coincidence that the tissue obtained by needle biopsy was taken from this region twice, LGESS could not be suspected preoperatively, and uterine leiomyoma was diagnosed due to these results, thus laparoscopic surgery was performed.

It is difficult to distinguish between LGESS and other types of tumors, such as leiomyoma with degeneration or cellular leiomyoma by MRI examination before surgery. Therefore, it is not uncommon for LGESS to be identified by postoperative pathological diagnosis after hysterectomy or tumorectomy for preoperative diagnosis of uterine leiomyoma, and there are reports of LGESS being diagnosed during/after laparoscopic surgery (6-8). When laparoscopic surgery is performed on LGESS, the risk of intraperitoneal dissemination should be taken into consideration. There are reports describing no recurrence after surgery, even when the tumor or uterus was divided prior to excision, but regarding the cases in which hysterectomy was performed with diagnosis of uterine leiomyoma, and finally diagnosed as LGESS, it has been reported that the recurrence rate is significantly higher in the divided group in which the tumor was excised separately for laparoscopic or vaginal surgery compared with the group in which the tumor was excised without division (9). If possible, it is desirable to avoid tumorectomy or division of the uterus for LGESS surgery, and for that purpose, a method for preoperative diagnosis of LGESS is required.

In the surgically resected specimen in the present case, the immunostaining findings showed positive staining for CD10 and negative staining for SMA in the region showing a typical LGESS section, but both SMA and CD10 were positive in the region where the LGESS was present with smooth muscle differentiation. Apart from this case, we have experienced 2 patients of uterine leiomyoma in which CD10 was partially positive. Therefore, a tumor that was morphologically suspected to be leiomyoma and positive for CD10 immunostaining cannot always be diagnosed as endometrial stromal tumor with smooth muscle differentiation. As a 
A

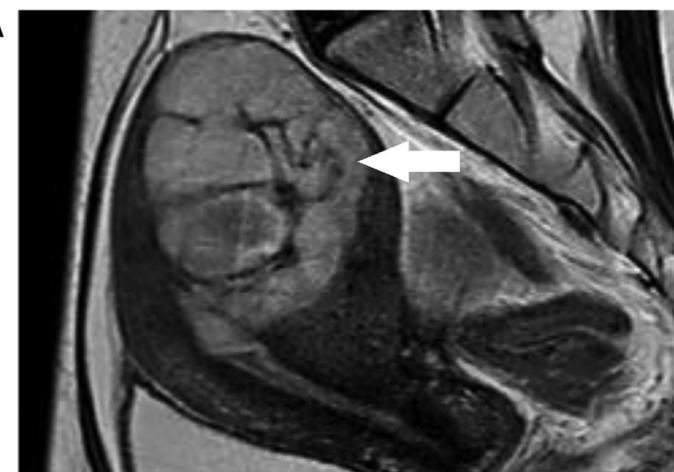

$\mathrm{C}$

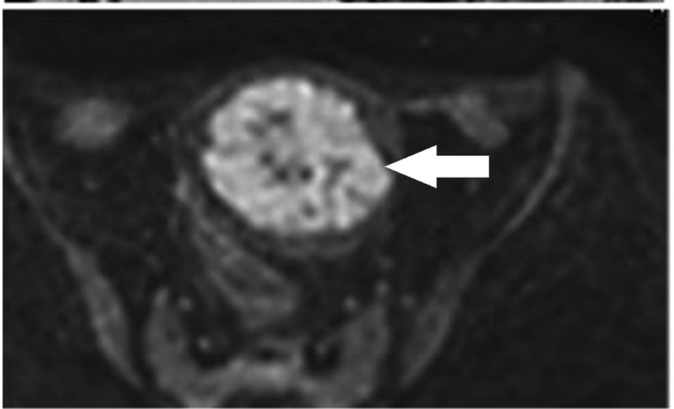

$\mathrm{B}$

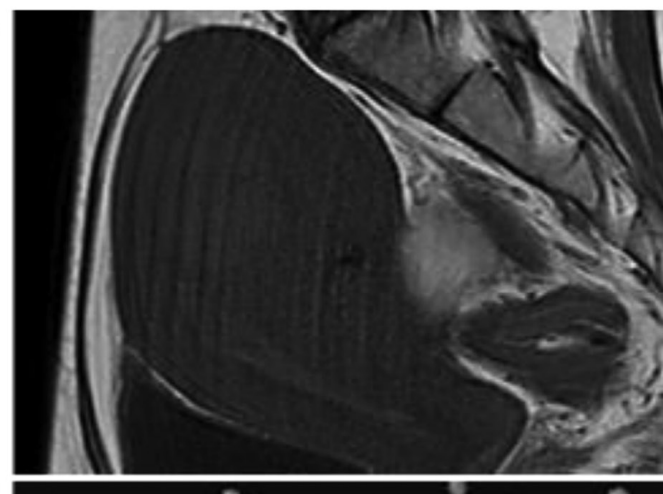

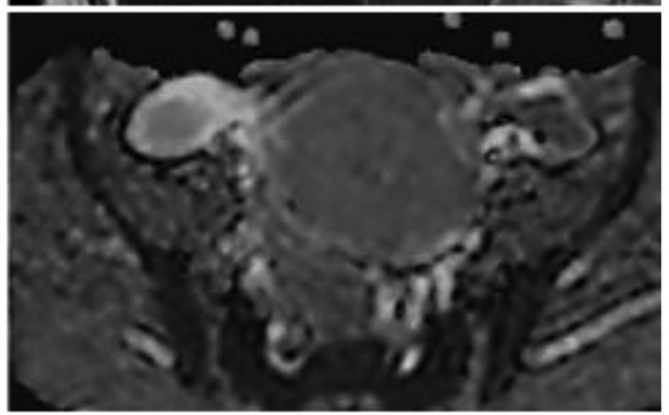

Figure 3. Magnetic resonance imaging scan 29 months after the first visit. The uterine tumor had grown in size to $68 \mathrm{x} 55 \mathrm{~mm}$. (A) T2-enhanced sagittal image: The tumor showed diffuse hyperintensity. Tumor is indicated by a white arrow. (B) T1-enhanced sagittal image: The tumor showed hypo-intensity. (C) Diffusion weighted image: The tumor showed hyper-intensity. Tumor is indicated by a white arrow. (D) ADC map: The ADC map showed an equivalent signal, no ADC degradation was observed, and it was considered that there was no diffusion limitation. ADC, apparent diffusion coefficient.
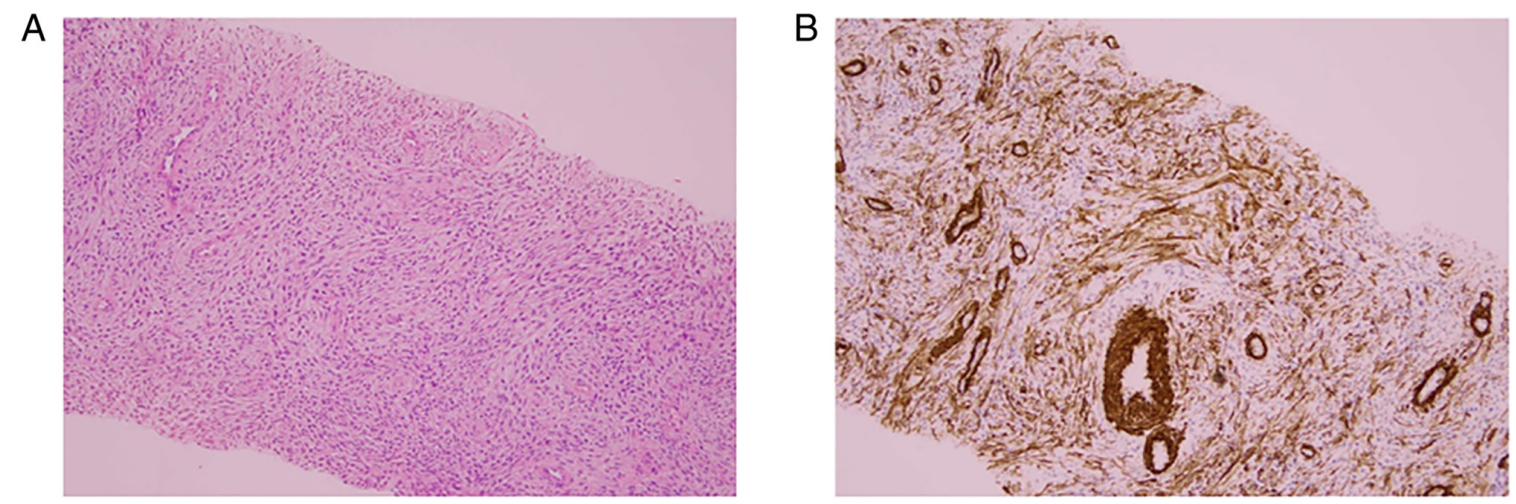

Figure 4. Microscopic image of the second needle-biopsied specimen. Pathological findings were similar to the first diagnosis (A) H\&E-stained specimen. (B) SMA immunostained specimen. Magnification, x100. SMA, smooth muscle actin.

retrospective analysis, CD10 immunostaining on the second needle biopsied specimen was performed, and it was shown that it too was also CD10 positive (Fig. 6A and B). When this CD10-immunostained section was observed in detail and compared with SMA-immunostaining of the same sample, a region where only CD10 was positive was observed. There are no reports of a uterine leiomyoma in which there is a region where SMA is negative but CD10 is positive, to the best of our knowledge. If SMA and CD10 immunostaining is performed on needle-biopsied specimen reliably collected from uterine tumors, and a region shows positivity only for CD10, even if the H\&E-stained specimen does not show a typical endometrial stromal tumor, it can be inferred that the tumor is not degenerative leiomyoma, but instead an endometrial stromal tumor. It is a well-known fact that CD10 immunostaining findings are useful information in the diagnosis of LGESS. In addition, several other papers have been reported on the differentiation between endometrial stromal sarcoma and leiomyoma based on immunostaining findings. Busca et al reported that IFITM1 (interferon-induced transmembrane protein-1) and CD10 had good sensitivity in differentiating between LGESS and smooth muscle tumor (10), and Zhu et al reported that a panel of h-caldesmon, CD10 and CD44v3 was most useful in differentiating endometrial stromal sarcoma from cellular leiomyoma (11). Furthermore, Zhao et al reported that the combination of IFITM1, CD10, SMA, and h-caldesmon was useful in distinguishing between endometrial stromal tumor and cellular leiomyoma (12). However, all of these reports are evaluations of surgically resected specimens, and the purpose of the study is to make final postoperative pathological diagnosis. On the other hand, although our report is a retrospective study, it is a report aimed at preoperative diagnosis 

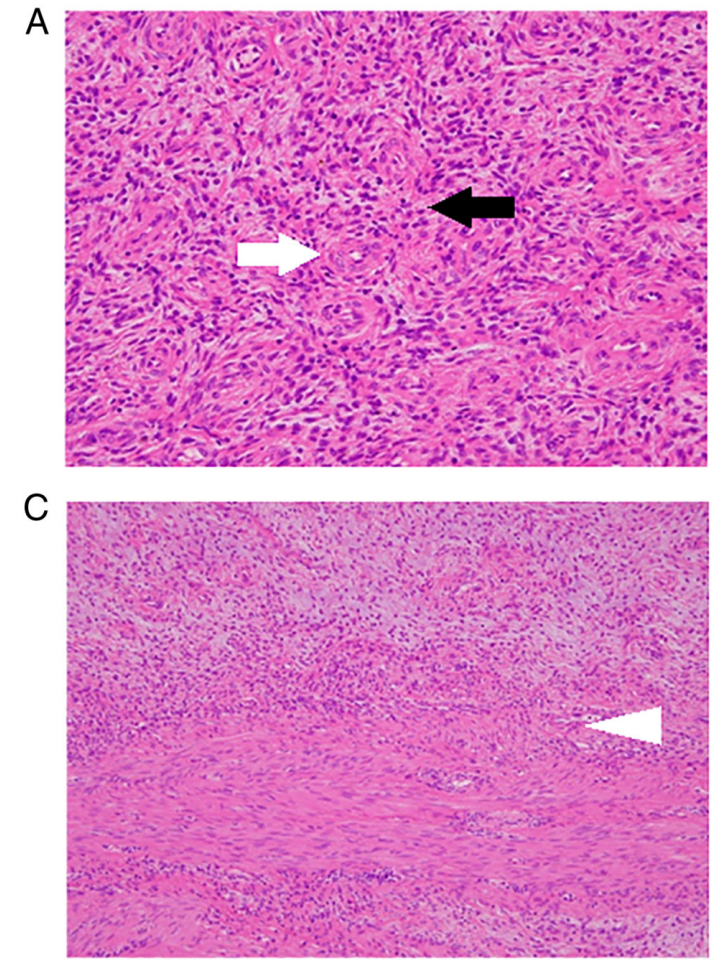

B

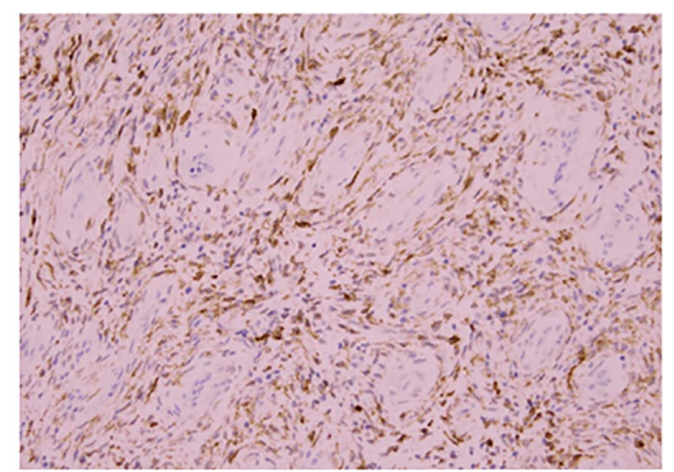

D

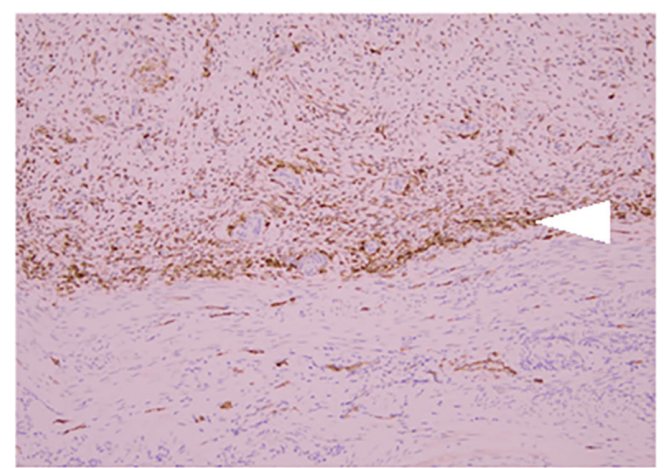

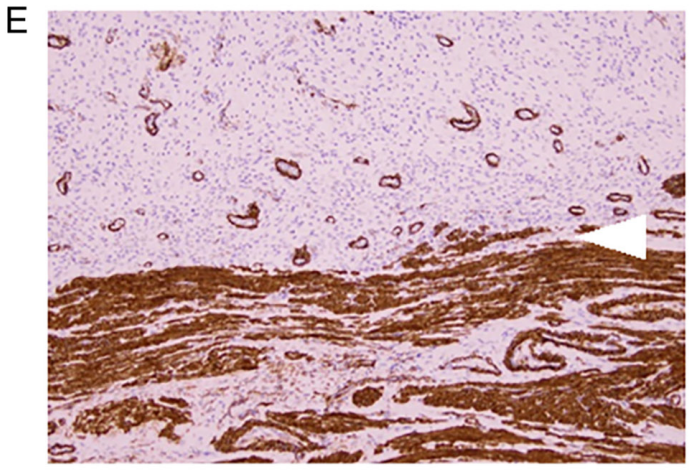

Figure 5. Microscopic images of the surgical specimen. (A) H\&E-stained specimen. Elliptical, relatively small tumor cells grew densely in a swirling pattern around the arterioles. No nuclear abnormalities or mitotic characteristics were observed. The black arrow indicates tumor cells that grow spirally around the arterioles and the white arrow indicates arterioles. (B) CD10-immunostained specimen; CD10 was diffusely positive. (C) Front formation of areas where oval and relatively small tumor cells proliferated (upper half) and areas where tumor cells with spindle-shaped nuclei proliferated (lower half). H\&E-stained specimen. (D and E) The upper half of the image consisted of CD10 positive/SMA negative cells, and the lower half consisted of CD10 negative/SMA positive cells. Differences in immunostaining results were clear. The white arrowhead indicates the front part (the front part refers to the boundary between the growth area of small tumor cells and the growth area of tumor cells with spindle-shaped nuclei). (D) CD10-immunostained specimen. (E) SMA-immunostained specimen. (A and B) Magnification, x200. (C-E) Magnification, x100. SMA, smooth muscle actin.
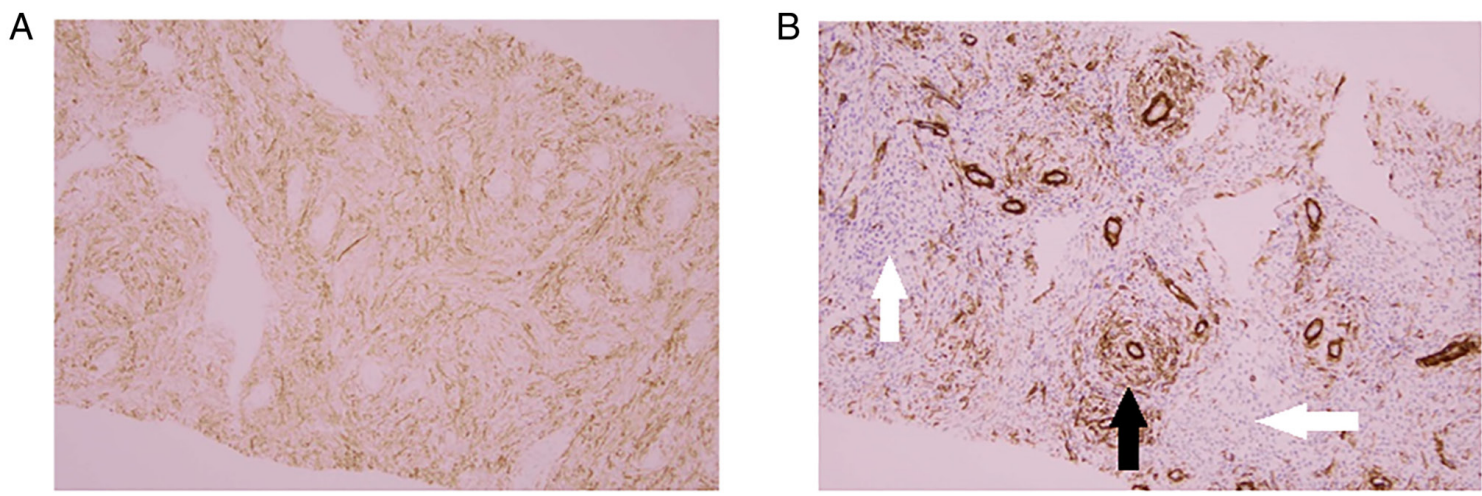

Figure 6. Microscopic image of the second needle-biopsied specimen. (A) CD10 immunostained specimen. CD10 was diffusely positive. (B) SMA immunostained specimen in the same region as (A) Blood vessels were positive for SMA, but the tumor was negative for SMA, even in the CD10-positive tumor region. The black arrow indicates blood vessels that are SMA positive and the white arrows indicate tumor areas where SMA is negative. Magnification, $\mathrm{x} 100$. SMA, smooth muscle actin. 
by immunohistological evaluation for biopsy specimens. The results of this study may be applicable to preoperative diagnosis, unlike previous reports. We believe that needle biopsy procedure and CD10/SMA immunostaining may establish a preoperative diagnosis for LGESS. This attempt is a new initiative that has never been reported.

In conclusion, a case of LGESS that was diagnosed as leiomyoma by needle biopsy prior to surgery by laparoscopic simple total hysterectomy is described. Since LGESS is relatively more common in younger individuals, laparoscopic surgery is likely to be performed if the preoperative diagnosis is leiomyoma, but laparoscopic tumorectomy may increase the risk of recurrence. It is suggested that immunohistochemical examination using anti-SMA and anti-CD10 antibodies on specimen obtained from needle biopsy may allow for preoperative diagnosis of LGESS including cases in which smooth muscle differentiation is present.

\section{Acknowledgements}

Not applicable.

\section{Funding}

No funding was received.

\section{Availability of data and materials}

The datasets used and/or analyzed during the current study are available from the corresponding author on reasonable request.

\section{Authors' contributions}

TI, MK and TS conceived and designed this case report. TI and TS wrote the initial draft. KI, MY, TF and TY collected clinical data. TI, MK and KI analyzed the data from the pathological images. TI, MK and TS confirmed the authenticity of all the raw data. All authors read and approved the final manuscript.

\section{Ethics approval and consent to participate}

Written informed consent was obtained from the patient prior to surgery and tissue collection.

\section{Patient consent for publication}

Written informed consent was obtained from the patient for the publication of this paper.

\section{Competing interests}

The authors declare that they have no competing interests.

\section{References}

1. Koss LG, Spiro RH and Brunschwig A: Endometrial stromal sarcoma. Surg Gynecol Obstet 121: 531-537, 1965.

2. Paul PG, Rengaraj V, Das T, Garg R, Thomas M and Khurd AS: Uterine sarcomas in patients undergoing surgery for presumed leiomyomas: 10 Years' Experience. J Minim Invasive Gynecol 23: 384-389, 2016.

3. WHO Classification of Tumours of Female Reproductive Organs. 4th edition. 2014.

4. Oliva E, Clement PB, Young RH and Scully RE: Mixed endometrial stromal and smooth muscle tumors of the uterus: A clinicopathologic study of 15 cases. Am J Surg Pathol 22: 997-1005, 1998.

5. Kawamura N, Ichimura T, Ito F, Shibata S, Takahashi K, Tsujimura A, Ishiko O, Haba T, Wakasa K and Ogita S: Transcervical needle biopsy for the differential diagnosis between uterine sarcoma and leiomyoma. Cancer 94: 1713-1720, 2002.

6. Della Badia C and Karini H: Endometrial stromal sarcoma diagnosed after uterine morcellation in laparoscopic supracervical hysterectomy. J Minim Invasive Gynecol 17: 791-793, 2010.

7. Takeda T, Tamada Y, Matoba Y, Saotome K and Makabe T: Low-grade endometrial stromal sarcoma which relapsed a month after transcervical resection as fertility-sparing surgery and diagnosed with laparoscopic hysterectomy. J Gynecol Fertil 1: 1-7, 2016.

8. Zheng Y, Yin Q, Yang X and Dong R: Fertility-sparing management of low-grade endometrial stromal sarcoma: Analysis of an institutional series, a population-based analysis and review of the literature. Ann Transl Med 8: 1358, 2020.

9. Park JY, Kim DY, Kim JH, Kim YM, Kim YT and Nam JH: The impact of tumor morcellation during surgery on the outcomes of patients with apparently early low-grade endometrial stromal sarcoma of the uterus. Ann Surg Oncol 18: 3453-3461, 2011.

10. Busca A, Gulavita P, Parra-Herran C and Islam S: IFITM1 Outperforms CD10 in differentiating low-grade endometrial stromal sarcomas from smooth muscle neoplasms of the uterus. Int J Gynecol Pathol 37: 372-378, 2018.

11. Zhu XQ, Shi YF, Cheng XD, Zhao CL and Wu YZ: Immunohistochemical markers in differential diagnosis of endometrial stromal sarcoma and cellular leiomyoma. Gynecol Oncol 92: 71-79, 2004.

12. Zhao W, Cui M, Zhang R, Shen X, Xiong X, Ji X, Tao L, Jia W, Pang L, Sun Z, et al: IFITM1, CD10, SMA, and h-caldesmon as a helpful combination in differential diagnosis between endometrial stromal tumor and cellular leiomyoma. BMC Cancer 21: $1047,2021$. 